A N N A L E S Annales de Bretagne et des Pays de l'Ouest

Anjou. Maine. Poitou-Charente. Touraine

110-4 | 2003

Espace et histoire

\title{
De la cotisation à la prime d'assurance
}

Le secours mutuel au Québec entre 1880 et 1945

Yvan Rousseau

\section{(2) OpenEdition}

Journals

Édition électronique

URL : http://journals.openedition.org/abpo/1348

DOI : $10.4000 /$ abpo. 1348

ISBN : 978-2-7535-1492-8

ISSN : 2108-6443

Éditeur

Presses universitaires de Rennes

\section{Édition imprimée}

Date de publication : 20 décembre 2003

Pagination : 151-169

ISBN : 978-2-86847-933-4

ISSN : $0399-0826$

Référence électronique

Yvan Rousseau, "De la cotisation à la prime d'assurance », Annales de Bretagne et des Pays de l'Ouest [En ligne], 110-4 | 2003, mis en ligne le 20 décembre 2005, consulté le 20 avril 2019. URL : http:// journals.openedition.org/abpo/1348; DOI : 10.4000/abpo.1348 


\title{
De la cotisation à la prime d'assurance Le secours mutuel au Québec entre 1880 et $1945^{1}$
}

\author{
Yvan RousSEAU \\ Professeur d'Histoire contemporaine \\ Centre interuniversitaire d'études québécoises \\ Université du Québec à Trois-Rivières \\ (avec la collaboration d'Annie DesAuLNIERS et Amélie MAINVILLE)
}

Vers la fin du $18^{\mathrm{e}}$ siècle, apparaissent les premières formes organisées de secours mutuel au Canada. Grâce aux cotisations de leurs membres, elles versent des indemnités en cas de maladie ou d'accident, offrent de petites sommes pour les funérailles et assistent les veuves et les orphelins. Le dessein de leurs promoteurs est de substituer à l'assistance des institutions destinées à cultiver le sens de la prévoyance parmi les classes populaires et à les responsabiliser à l'égard des aléas de la vie. Jusqu'à ce que leur existence soit légalement reconnue en 1850 par le gouvernement du Canada-Uni ${ }^{2}$, ces petites associations surgissent ici et là dans les zones les plus industrialisées, puis connaissent le plus souvent une existence précaire, sinon éphémère. En levant partiellement le climat de suspicion qui prévalait à leur égard, la loi de 1850 va encourager leur multiplication dans la seconde moitié du $19^{\mathrm{e}}$ siècle, sans toutefois changer grand-chose à leur précarité financière. Comme on n'oblige pas au départ les sociétés à s'enregistrer auprès des pouvoirs publics, elles ont laissé peu de traces et il est très difficile d'évaluer l'ampleur du mouvement ${ }^{3}$. Les estimations les

1. Nos remerciements vont au Conseil de la recherche en sciences humaines qui a manifesté son appui à ce travail. Nous avons également une dette importante envers Marie-Ėve Lachapelle et Roxanne Martin qui nous ont assisté avec diligence dans le travail de dépouillement.

2. «Acte pour incorporer certaines associations charitables, philanthropiques et de prévoyance, et pour protéger d'une manière efficace les fonds des dites associations contre la fraude et le mauvais emploi qu'on en pourrait faire " (13-14 Vict. [1850], chap. 32).

3. Pour s'en donner une idée, il suffit de lire ces quelques lignes d'une longue lettre de l'inspecteur provincial des sociétés de secours mutuel au Secrétaire de la Province, faisant office de rapport annuel en $1902:$ « [...] Les Sociétés de Secours Mutuels et Associations Charitables, constituées dans la Province [...], n'attachent aucune 
plus prudentes font état de la création d'une centaine de ces sociétés de secours mutuel au $19^{\mathrm{e}}$ siècle, mais il est vraisemblable qu'elles aient été bien plus nombreuses ${ }^{4}$. Elles s'établissent principalement à Montréal, à Québec et dans les petits centres industriels qui essaiment un peu partout à cette époque.

Ce n'est pas le lieu ici d'analyser la dynamique et l'évolution de ces mutuelles de première génération, d'autres l'ont fait avant nous par des études de cas ou dans des perspectives plus générales ${ }^{5}$. Pour les besoins de notre propos, relevons certains de leurs traits prédominants. D'abord, elles entretiennent des liens privilégiés avec les communautés locales, où elles s'enchevêtrent aux solidarités de métiers, d'appartenances ethniques et de confessions religieuses. Si plusieurs présentent une certaine unité sur le plan idéologique ou sur celui des membres qu'elles desservent (comme les Unions Saint-Joseph ou les Sociétés Saint-Jean-Baptiste, par exemple), elles restent foncièrement autonomes sur le plan financier. Leur domaine de prédilection est la protection contre les aléas occasionnés par les accidents et la maladie ${ }^{6}$, même si un certain nombre d'entre elles transigent de façon accessoire une assurance pour les décès.

Ces sociétés sont par ailleurs le siège d'une vie associative particulièrement intense. Dans le contexte de milieux sociaux bouleversés par l'industrialisation, elles viennent exprimer les identités collectives et rappeler les valeurs communes qui les ont engendrées. Rituels initiatiques, organisation d'activités de loisir, participation aux cérémonies religieuses, représentation officielle aux funérailles, commémorations patriotiques, visites des malades et tenue de réunions régulières sont autant d'occasions de célébrer le "Nous " et d'évoquer les services qu'elles rendent à leurs membres. Toute cette activité culturelle, parce qu'elle se déploie locale-

importance à la loi, et ne s'y soumettent que quand bon leur semble. Chaque année, des blancs de rapports leur ont été envoyés, et quand elles prennent la peine de faire ces rapport, c'est avec une négligence impardonnable qu'elles le font [...]. „ Documents de la session (Québec), Rapport sur les sociétés de secours mutuels, Bureau du secrétaire, 1902 , p. 5 .

4. DeschêNEs, Gaston, "Associations coopératives et institutions similaires au $20^{\mathrm{e}}$ siècle ", Revue d'histoire de l'Amérique française, 29, 4 (mars 1976), p. 545. Pour notre part, nous avons recensé pas moins de 82 sociétés de secours mutuels ayant transigé des affaires au Québec avant 1900. Ce relevé ne considère que celles ayant survécu à l'année 1909, alors que le Surintendant des assurances du Québec commence à tenir avec plus de soin les statistiques sur leurs activités.

5. Pour une vue générale, on consultera PETITCLERC, Martin, "La protection familiale : les solutions capitaliste et mutualiste jusqu'en 1880 ", document ronéotypé (à paraître dans Saint-Pierre, Diane, Saint-Pierre, J. et Petitclerc, M., Histoire de l'assurance de personnes au Québec, des sociétés de secours mutuel aux grandes institutions d'assurance, Québec, Les Éditions de l'IQRC, 2002).

6. "I have found, écrit le premier inspecteur des assurances du Québec en 1884, over fifty Acts of Incorporation for such associations since Confederation, and the object of all seems to be pretty much the same, namely: "The aiding and assisting of members and their families in case of sickness by means of monthly payments" "Documents de la session (Québec), The Report of the Inspector of Insurance for the Province of Quebec, 1884, p. 13. 
ment et prend appui sur les réseaux d'interconnaissance, participe également d'une rationalité économique bien particulière. Alors que l'on ne dispose pas encore de tables actuarielles en matière d'assurance-maladie, connaître ses semblables et être connu de façon réciproque prend un sens précis et plein : cela permet en effet de garder à vue les malades bénéficiant des indemnités et d'écarter les demandeurs d'admission présentant trop de risques.

Au cours des années 1880-1900, le rythme de fondation des mutuelles locales s'essouffle et, au tournant du $20^{\mathrm{e}}$ siècle, elles entrent irrémédiablement dans leur phase de déclin. Entre 1914 et 1940, le nombre des sociétés de secours mutuel en activité au Québec passe en effet de 150 à 109 . $\mathrm{Au}$ cours de cette période, elles sont environ 140 à abandonner leurs affaires ${ }^{7}$. La plupart le font en raison d'insuffisances financières, plusieurs meurent dans l'œuf, tandis qu'une trentaine d'entre elles se sabordent pour joindre les rangs des grandes sociétés fraternelles.

Pourtant, l'adhésion aux sociétés de secours mutuel s'accroît de façon impressionnante entre 1880 et la Grande Guerre. En 1915, elles desservent au-delà de 175000 adhérents, dont plus de la moitié dans des établissements basés au Québec. C'est presque le double du nombre enregistré en 1900 et plus de cinq fois celui de 1880. Cette croissance sera toutefois beaucoup plus modérée pendant l'entre-deux-guerres, pendant que les sociétés québécoises vont accroître de façon significative leur influence : on recense 212000 adhérents en 1939 dont plus des trois quarts à des mutuelles québécoises ${ }^{8}$.

Parmi celles qui connaissent la croissance la plus vive, figurent deux sociétés fraternelles, la Société des artisans canadiens-français et l'Alliance nationale, que nous allons aborder après avoir dit quelques mots du mouvement plus large dans lequel elles s'insèrent.

\section{La poussée des sociétés fraternelles}

Tandis que le secours mutuel local parvient mal à assurer sa pérennité, une seconde génération de mutuelles gagne en popularité dans seconde moitié du $19^{\mathrm{e}}$ siècle : les grandes sociétés fraternelles. Originaires de Grande-Bretagne, elles gagnent les États-Unis dans le premier quart du $19^{\text {e }}$ siècle par l'intermédiaire des émigrants, puis la région de Montréal au cours des années 1840 alors que sont établies huit loges de l'Ordre des Odd Fellows, une société offrant une protection contre la maladie aux jeunes hommes de la bourgeoisie anglo-protestante. Plusieurs suivront dans le der-

7. Relevé personnel à partir d'un dépouillement exhaustif des Rapports annuels du surintendant des assurances du Québec, 1914-1941. Le taux de survie se situe bien en deçà de ces chiffres si l'on exclut les mutuelles d'employés et les grandes sociétés fraternelles qui procèdent de logiques quelque peu différentes.

8. Selon les chiffres fournis par le surintendant des assurances (Rapport annuel du surintendant des assurances du Québec, 1940). 
nier quart du $19^{\mathrm{e}}$ siècle, la plupart dirigeant leurs affaires depuis les ÉtatsUnis et l'Ontario. En dehors des deux ordres de Forestiers (Canadian Order of Foresters et Catholic Order of Foresters) et de l'Association canado-américaine qui connaissent une progression appréciable jusqu'aux années 1910, elles ne parviennent que difficilement à s'enraciner au Québec, notamment chez les Canadiens français. Il faut dire que les condamnations explicites de Rome en 1894 et l'ardeur avec laquelle le clergé catholique québécois les appliquent n'ont rien pour leur faciliter les choses ${ }^{9}$. En revanche, ce dernier se révèle un promoteur zélé, surtout à compter de Rerum Novarum, des mutuelles catholiques canadiennes-françaises.

Comme leurs devancières, les sociétés fraternelles offrent généralement une protection contre la maladie, mais plusieurs d'entre elles l'assortissent d'une obligation de souscrire à une assurance-vie, une activité à laquelle elles vont accorder une importance grandissante avec les années. Elles vont apporter des changements importants dans la mutualité d'assurance : 1) le recrutement à grande échelle (territoriale et professionnelle); 2) la coordination centrale des unités locales des membres (par des systèmes de loges, de cercles et de succursales); 3) l'adoption d'un mode contributoire basé sur les principes actuariels ${ }^{10}$; 4 ) le relâchement, surtout à partir des années 1900, des critères d'adhésion fondés sur la profession, la religion, l'ethnie ou le sexe. Comme les grandes compagnies d'assurance qui connaissent un essor phénoménal entre 1880 et 1915 , les sociétés fraternelles recherchent l'éventuel assuré qu'elles définissent comme membre plutôt que client. Pour les attirer dans leurs rangs, elles misent sur une offre de services à bon marché et font miroiter les avantages d'une participation démocratique des assurés à la gestion et au développement de l'association. Tout un arsenal de propagande va se charger de répandre le message mutualiste : publicité dans la grande presse, revues et brochures promotionnelles, embauche de propagandistes, etc. L'idée est de convaincre de la viabilité financière de la mutualité, de persuader que l'assurance constitue une forme d'épargne, qu'elle a des vertus morales et qu'elle repose sur des fondements scientifiques rigoureux.

9. En 1894, le pape interdit aux Catholiques, sous peine d'excommunication, de joindre les rangs des Odd Fellows, des Knights of Pythias, des Sons of Temperance. Outre ces trois sociétés, d'autres s'ajoutent dans la liste des organisations condamnées par le clergé catholique québécois en 1896, notamment : Canadian Order of Foresters, Grand Council of the Canadian Order of Chosen Friends, Subsidiary High Court of the Ancient Order of Foresters, Supreme Select Knights of Canada, Dominion Council of Canada and Newfoundland of Royal Templars of Temperance, Supreme Council of the Home Circle (PETITCLERC, op. cit.).

10. Ce sont ces ordres fraternels qui sont à l'origine de la création, en 1886, du National Fraternal Congress of America, une puissante organisation chargée en outre de promouvoir l'introduction de la gestion actuarielle au sein du secours mutuels. Ils jouent par ailleurs un rôle clé dans les modifications majeures apportées à la législation relative au secours mutuel par les États en Amérique du Nord au tournant du $20^{\mathrm{e}}$ siècle. 
Figure 1 - L'essor de l'assurance-vie au Québec et au Canada, 1875-1935 (primes d'assurance souscrites et nombre d'établissements en activité)* (Sources : relevé personnel à partir des Rapports annuels du Surintendant des assurances de la province de Québec et des Rapports annuels du Surintendant des assurances du Canada.)

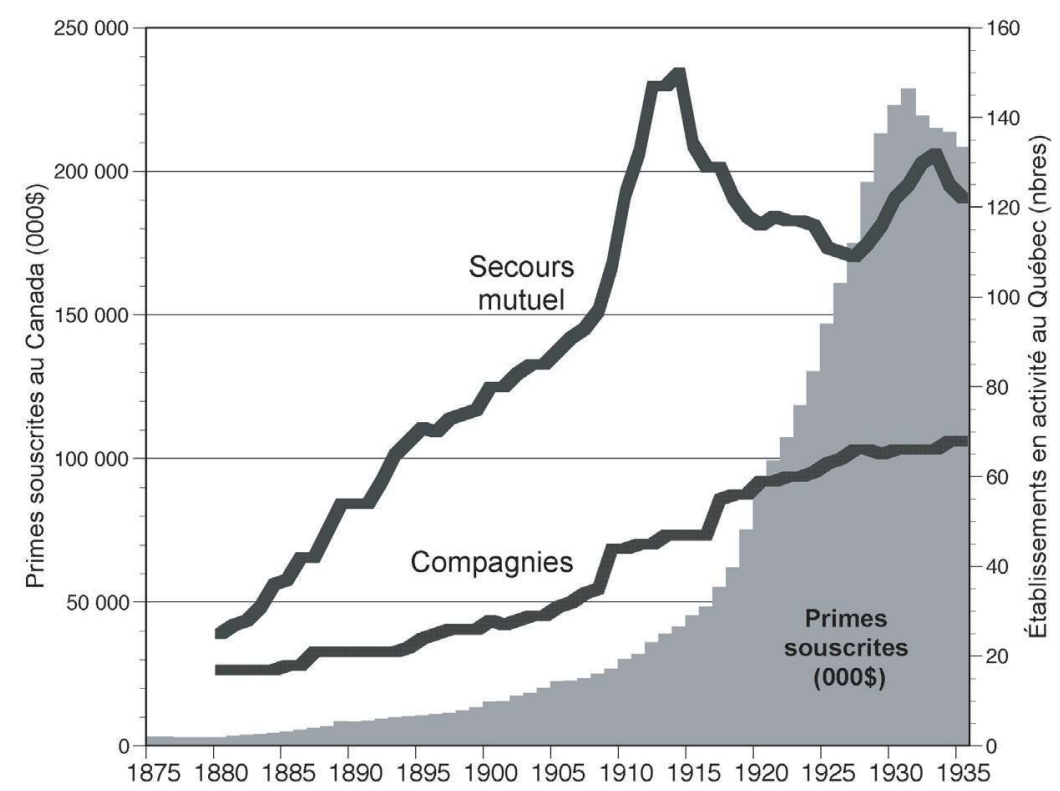

* Les chiffres relatifs au secours mutuel avant 1909 sont approximatifs.

Des relations privilégiées entretenues par le Canada avec l'empire britannique, la proximité des États-Unis et une situation culturelle singulière dans l'environnement nord-américain ont engendré au Québec la formation de deux configurations distinctes du monde de l'assurance, inégalement développées, renvoyant chacune à des réseaux d'acteurs, des marchés et des institutions qui leur sont propres. Cette dualité, qui recoupe partiellement les deux grands groupes linguistiques de la population québécoise, traduit également la présence de différences importantes dans les modes de diffusion de l'assurance et ses formes juridiques d'organisation. Ainsi, le Québec a engendré très peu de compagnies d'assurance-vie (par actions ou mutuelles) : entre 1880 et 1930, neuf sociétés d'assurance québécoises sur dix sont organisées en vertu du cadre juridique du secours mutuel. En revanche, il représente un marché de prédilection pour les grandes compagnies étrangères et anglo-canadiennes qui, au début de la Seconde Guerre, attirent vers elles au-delà de $92 \%$ des primes d'assurancevie versées par les Québécois ${ }^{11}$.

11. Nous avons traité cette question ailleurs : "Les marchés de l'assurance-vie au Québec entre 1880 et 1960 : une étude de la distribution spatiale des centres de décision 
La faiblesse des bases d'accumulation privée au Québec semble donc avoir encouragé la mise en place d'associations mutuelles d'assurance se définissant comme canadiennes-françaises ${ }^{12}$. Si elles font pâle figure comparées au gigantisme de leurs concurrentes de l'extérieur du Québec, leur rôle pendant l'entre-deux-guerres n'en est pas moins crucial dans l'évolution que vont connaître plus tard les marchés financiers québécois. Elles ont constitué, c'est notre hypothèse générale, des vecteurs privilégiés dans l'accumulation et la centralisation des capitaux, en même temps qu'elles ont contribué à la formation d'une élite de gestionnaires rompus aux manières de faire des grandes firmes d'assurance capitalistes. Cette réorientation de la mutualité, il va sans dire, est l'objet et l'enjeu de tensions récurrentes entre partisans de la centralisation et promoteurs de l'autonomie locale. Elle tient moins aux mouvements d'idées (qui présentent une étonnante continuité), qu'à leur rapport au territoire, à l'assise sociale de leur association et à leur mode de fonctionnement.

Bref, c'est tout le rapport historiquement constitué entre les mutualistes et leurs organisations de base qui, avec le concours du service des assurances de l'État québécois, se redéfinit pendant l'entre-deux-guerres. Le cas des Artisans canadiens-français et de l'Alliance nationale, que nous allons maintenant prendre à témoin, offre un éclairage intéressant sur les grands processus qui secouent les sociétés fraternelles au cours de cette époque.

\section{Les territoires de la mutualité canadienne-française}

Les Artisans canadiens-français et l'Alliance nationale, comme leurs noms le suggèrent, s'adressent expressément aux collectivités canadiennesfrançaises, à qui elles offrent une assurance-vie assortie d'une protection contre la maladie. La première est fondée en 1876 sous le nom de Société des Artisans canadiens-français de la cité de Montréal par un groupe de petits entrepreneurs et d'ouvriers de métiers qui souhaitent ouvrir les rangs de leur association à " toutes les professions commerciales, industrielles et manuelles, réputées non insalubres ${ }^{13}$ ". Pour surmonter les difficultés financières que connaissent la plupart des sociétés de secours mutuel locales avec leurs caisses de maladie, on insiste sur la constitution de réserves, mais l'on conserve le système de cotisation égalitaire qui pré-

et de l'appropriation des revenus ", BellaVAnCE, Claude et LANTHIER, Pierre (dir.), Les Territoires de l'entreprise, à paraître; "Les réseaux d'assureurs au Québec entre 1900 et 1960 : une analyse sociographique ", Des francophonies périphériques, Louvain-la-Neuve, 13-15 mai 2003.

12. À la manière de la situation que nous avons analysée dans le cas du secteur bancaire. À ce sujet : en collab. avec LEVASSEUR, Roger, "Social Movement and Development in Quebec. The Experience of the Desjardins Movement from 1900 to the End of the 1960s ", Annals of Public and Cooperative Economics, 72, 4, 2001, p. 549-579.

13. Constitution et règlements de la Société des artisans canadiens-français de la cité de Montréal, Montréal, Imprimerie de Louis Perreault et $C^{\text {ie }}, 1877$. Il faudra attendre 1906 avant que l'on introduise les tables actuarielles pour fixer les cotisations. 
vaut alors un peu partout ${ }^{14}$. La forte concurrence sur le marché montréalais et les percées effectuées au Québec par les fraternelles états-uniennes et ontariennes l'amènent en 1889 à envisager une expansion vers l'extérieur de Montréal. Des unités " locales " seront d'abord organisées à Lévis et à Québec, puis dans les petites villes industrielles du sud-ouest du Québec. Suivent, en 1894, une première succursale à Ottawa (en Ontario) et, trois ans plus tard, une dizaine de succursales franco-américaines.

L'Alliance nationale voit le jour alors même que la Société des Artisans se lance dans le développement de son réseau de succursales. En 1892, en effet, une centaine de Montréalais fondent une société de secours mutuel à peu près similaire à celle des Artisans. Le profil social des signataires de la déclaration de fondation donne toutefois des indications précieuses sur l'orientation plus affairiste qui sera celle de l'Alliance : plus de la moitié d'entre eux sont des hommes d'affaires, le quart appartient aux professions libérales et plusieurs autres dirigent des établissements publics ou privés ${ }^{15}$. Parmi ces fondateurs, figurent des représentants éminents du monde politique francophone de Montréal. Leur projet est de soustraire les Canadiens français à l'influence qu'exercent alors les compagnies d'assurance-vie et les fraternelles étrangères et, partant, de constituer un capital en vue de financer l'entreprise et le commerce canadiens-français. Par l'emploi des méthodes actuarielles, on souhaite offrir aux Catholiques de langue française une entreprise moderne " afin de pouvoir faire compétition avec avantage aux sociétés étrangères ${ }^{16}$ ". Comme les Artisans, l'Alliance préconise la mise en place d'un système de succursales à base paroissiale qu'elle baptise « cercles ». Très rapidement, elle va déployer son organisation vers l'extérieur de Montréal : dans les centres industriels québécois d'abord, puis vers les campagnes et les villes d'accueil des francophones dans les états du nord-est des États-Unis et du Canada anglais.

Au total, voilà donc deux sociétés fraternelles dont les similarités sont pour le moins intrigantes : toutes les deux dirigent leurs activités depuis Montréal, leur offre de services et leurs organisations sont à peu près semblables, leur discours interpelle les classes populaires qu'on veut rompre aux habitudes de prévoyance par la petite épargne, et elles se présentent enfin comme des leviers d'émancipation économique des Canadiens français. Ajoutons à ceci une correspondance à peu près parfaite de leurs cycles de développement.

En effet, la croissance des Artisans et de l'Alliance sera impressionnante et, en peu de temps, elles acquièrent la stature de véritables institutions natio-

14. C'est-à-dire, un mode contributoire fixant les cotisations sans égard à l'âge des assurés.

15. DESAULNIERS, Annie, Le Secours mutuel en transformation : l'Alliance nationale (18921948), Université du Québec à Trois-Rivières (M. A. Études québécoises), 2002, p. 32-37.

16. Propos de Mgr Fabre, archevêque de Montréal, qui aurait joué un rôle important dans la création de la société. "L'Alliance nationale, société de bienfaisance catholique de langue française ", L'Alliance nationale, 21, 8 (août 1915), p. 100. 
nales : en 1930, les deux organisations réunies reposent sur un réseau d'environ 1300 succursales locales (700 pour les Artisans, 620 pour l'Alliance) et rassemblent près de 100000 membres (respectivement 60000 et 40000 membres adultes). En l'espace d'une quinzaine d'années, elles sont parvenues à se hisser en tête de peloton des sociétés fraternelles au Québec, loin devant leurs concurrentes des États-Unis et de l'Ontario avec qui elles rivalisaient en 1915 avec des parts de marché à près égales. C'est cette expansion soutenue des fraternelles canadiennes-françaises, bien davantage que l'essor des compagnies commerciales à la même époque, qui précipite le déclin du secours mutuel local que nous avons relevé plus tôt. Parce qu'il exerce un effet de blocage sur les possibilités d'éclosion de nouvelles associations, le déploiement de ces réseaux de succursales est au cœur d'une importante vague de concentration de la mutualité québécoise. Ajoutons de surcroît que plus d'une vingtaine de sociétés, en majorité des Unions SaintJoseph, sont absorbées entre 1918 et 1929 par les deux fraternelles ${ }^{17}$.

Figure 2 - Répartition des membres des Artisans canadiens-français en 1906 (nombre d'adhérents par localité) (Sources : Rapports annuels, rapports des conventions générales, revue L'Alliance nationale.)

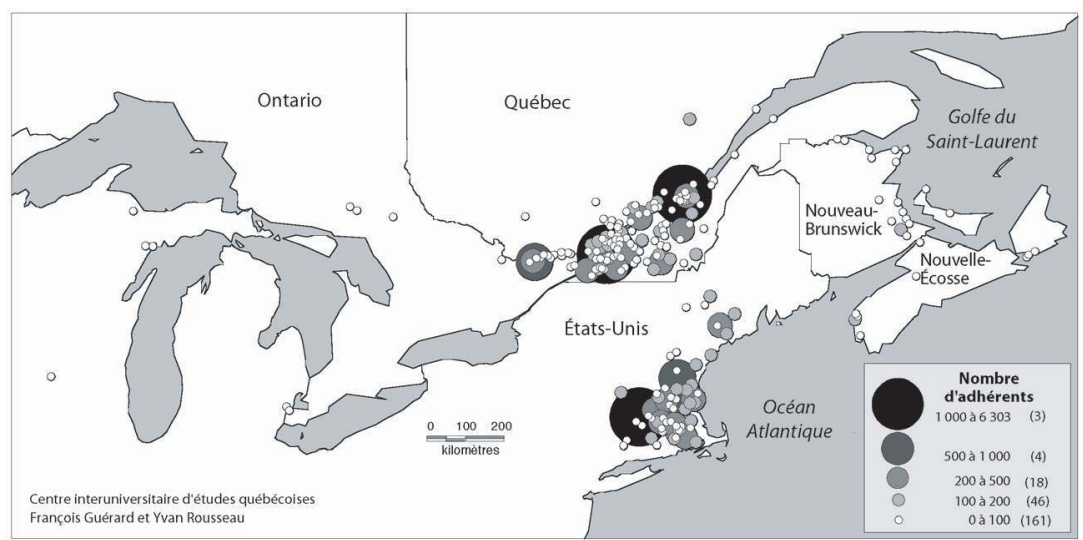

L'analyse comparative de la répartition spatiale des effectifs des deux fraternelles canadiennes-françaises en 1906 fournit des indications précieuses sur ce qui apparaît comme des traits distinctifs des deux organisations. À l'époque, le nombre de succursales des Artisans se chiffre à 295, tandis que l'Alliance en compte 282. La première présente d'abord un caractère urbain très affirmé, résultat de ses efforts pour desservir les populations de cols bleus des zones les plus industrialisées (fig. 2). Trois grandes aires d'influence ressortent à l'examen de la carte : Montréal, Québec et la côte est des États-Unis. Remarquons par ailleurs la présence d'une qua-

17. À ce sujet, voir notre article : « Les marchés de l'assurance-vie au Québec entre 1880 et $1960 \ldots$ ". op. cit. 
Figure 3 - Répartition des membres de l'Alliance nationale en 1906 (nombre d'adhérents par localité) (Sources : Rapports annuels, rapports des conventions générales, revue L’Artisan.)

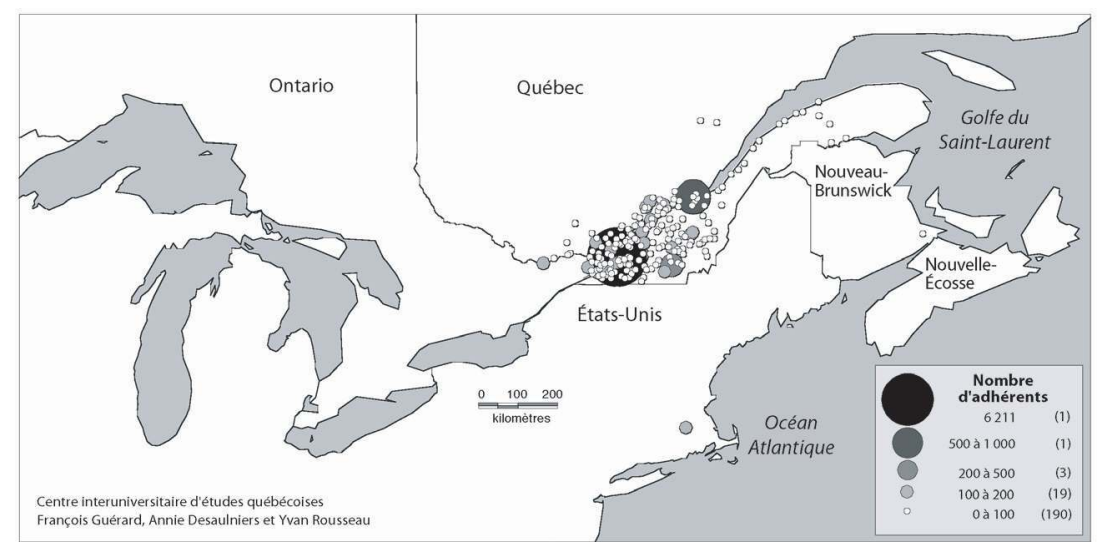

rantaine de succursales desservant les communautés francophones des Maritimes et de l'Ontario. En comparaison, l'Alliance nationale apparaît comme la plus "québécoise " des deux mutuelles (fig. 3). C'est au Québec qu'elle réalise d'ailleurs l'essentiel de ses activités, dont l'importance relative fluctue bon an mal an autour de $90 \%$ de son volume d'affaires global. En comparaison, le Québec représente entre 60 et $65 \%$ du marché des Artisans, une proportion qui se maintient jusqu'à la fin de la Seconde Guerre. Plus "québécoise ", l'Alliance apparaît également comme la plus " montréalaise " des deux sociétés : elle recrute en effet $40 \%$ de ses adhérents à Montréal, tandis que les Artisans le font dans une proportion de $20 \%$. La cartographie attire enfin l'attention sur les efforts déployés par l'Alliance pour s'implanter dans les milieux ruraux, un peu comme si la présence de sa concurrente dans les zones industrielles la repoussait en quelque sorte vers les campagnes. Aussi n'est-il pas étonnant que ses succursales soient généralement de taille plus modeste que celles des Artisans : 59 membres en moyenne, en comparaison de 109 pour la seconde.

Maintenant que nous avons esquissé à larges traits les particularités de l'assise territoriale de chacun des deux réseaux de succursales, approchons-nous des hommes qui les animent.

\section{Ouvriers et petits bourgeois dans le mouvement mutualiste}

Ouvriers, les membres des Artisans et de l'Alliance? Ils représentent une proportion de plus en plus consistante de leurs assurés, mais le moins que l'on puisse dire, c'est que leur présence à la direction des différentes unités de direction du mouvement reste discrète. Entre 1895 et 1945, en effet, le sociétariat des deux sociétés s'élargit pour accueillir en plus grand nombre de membres en provenance des couches inférieures de la classe ouvrière. 
Parallèlement, les critères d'admissibilité s'élargissent progressivement pour inclure les femmes, les plus âgés et les enfants ${ }^{18}$, en même temps que se diversifie l'offre de services ${ }^{19}$. L'extension de l'offre de services dans les deux mutuelles n'est pas sans rapport avec cette diversification de leur assise sociale. L'examen du profil socio-professionnel de leurs membres et dirigeants va nous fournir quelques clés d'explication dans ce sens.

Voyons d'abord le cas des membres. La revue L'artisan publie régulièrement des informations sur les professions des membres (les membres actifs, comme ceux qui décèdent), de sorte qu'il est possible de se faire une bonne idée du profil général de ces derniers. Malheureusement, on ne trouve pas d'équivalent à l'Alliance nationale. On devra donc restreindre l'examen du profil des membres aux seuls Artisans et présumer de l'existence de phénomènes relativement semblables chez sa concurrente.

Tableau 1 - Profil socio-professionnel des membres des Artisans canadiens-français, 1904 et selon deux périodes d'adhésion (nombres et \%)*

(Sources : Compilation personnelle à partir des informations livrées dans

L'Artisan et dans le Rapport de la quatrième convention de la Société des Artisans canadiens-français tenue à Montréal les 4-5-6 et 7 juillet 1904, Montréal, Arbour et Laperle, 1904, 200 p.)

\begin{tabular}{|c|c|c|c|c|c|c|}
\hline \multirow[b]{2}{*}{ Catégories socio-professionnelles } & \multicolumn{2}{|c|}{ en 1904} & \multicolumn{2}{|c|}{ avant 1889} & \multicolumn{2}{|c|}{ après 1888} \\
\hline & Nbre & $\%$ & Nbre & $\%$ & Nbre & $\%$ \\
\hline Manufacturiers/industriels & 111 & 0,6 & 2 & 1,0 & 1 & 0,6 \\
\hline Marchands et artisans & 4435 & 23,0 & 75 & 37,3 & 31 & 17,6 \\
\hline $\begin{array}{l}\text { Professions libérales et } \\
\text { personnel hautement qualifié }\end{array}$ & 1017 & 5,3 & 14 & 7,0 & 16 & 9,1 \\
\hline $\begin{array}{l}\text { Personnel dirigeant des } \\
\text { administrations et entreprises }\end{array}$ & 230 & 1,2 & 10 & 5,0 & 1 & 0,6 \\
\hline Cols blancs semi et non qualifiés & 2049 & 10,6 & 34 & 16,9 & 3 & 1,7 \\
\hline Cultivateurs & 1855 & 9,6 & 28 & 13,9 & - & - \\
\hline $\begin{array}{l}\text { Cols bleus qualifiés } \\
\text { et ouvrier de métier }\end{array}$ & 3722 & 19,3 & 4 & 2,0 & 44 & 25,0 \\
\hline $\begin{array}{l}\text { Cols bleus semi } \\
\text { et non qualifiés }\end{array}$ & 5896 & 30,5 & 34 & 16,9 & 80 & 45,5 \\
\hline Indéterminés et inclassables & 185 & - & 1 & - & 22 & - \\
\hline Total & 19500 & 100,0 & 202 & 100,0 & 198 & 100,0 \\
\hline
\end{tabular}

* Les professions indéterminées ne sont pas considérées dans le calcul des pourcentages.

18. Seuls les hommes âgés de 18 à 45 ans chez les Artisans et de 18 à 55 ans à l'Alliance sont admissibles au départ. Entre 1906 et 1915, les deux mutuelles ajustent graduellement leurs critères à ceux des fraternelles états-uniennes pour englober les individus âgés entre 16 et 60 ans. Les Artisans commencent à admettre les femmes en 1906, tandis que l'Alliance ne le fera qu'en 1913, au terme de virulents débats. En 1915, les premiers commencent à émettre des certificats à l'intention des enfants. L'Alliance emboîte le pas quatre ans plus tard.

19. En 1902, les Artisans n'émettent que des polices de $1000 \$$, tandis que sa concurrente le fait pour des montants allant de 500 à $3000 \$$. En 1918, la valeur des certificats varie de 100 à $5000 \$$ chez les premiers et de 250 à $5000 \$$ chez la seconde. 
Les chiffres de 1904 (tableau 1), que nous avons retenus dans un premier temps, traduisent la présence d'un sociétariat relativement diversifié : la moitié des membres des Artisans sont recrutés parmi les cols bleus, en particulier chez les plus qualifiés d'entre eux. L'autre groupe dont le poids est loin d'être négligeable est formé de petits commerçants et d'artisans qui forment à eux seuls près du quart des membres de la fraternelle. On retrouve ici principalement des épiciers, des bouchers et des boutiquiers pour la plupart (60\% du groupe), mais aussi une proportion non négligeable de membres exerçant des métiers artisanaux comme forgerons, cordonniers, selliers, etc. Si ces chiffres expriment l'enracinement de la société parmi le monde ouvrier, ils nous laissent cependant avec une image un peu statique des grands processus à l'œuvre au sein de la mutualité d'assurance. En séparant cette fois-ci les membres selon la période d'adhésion, on aperçoit très nettement cet élargissement des bases sociales du sociétariat de l'association vers les groupes évoluant aux échelons inférieurs de l'organisation du travail. La proportion des cols bleus est ainsi portée de 19 à $70 \%$, tandis que celle des marchands et des artisans, groupes d'appui de première souche du mouvement, évolue en sens inverse : $37 \%$ des adhérents avant 1889 en comparaison de $18 \%$ pour la période suivante. Le recul radical des cultivateurs et des cols blancs au cours des deux périodes reste énigmatique. Il peut s'agir d'une déficience des données, mais il peut également s'expliquer au moins partiellement par la concurrence de l'Alliance nationale, dont on a relevé précédemment les efforts pour s'implanter en milieu rural et s'attirer la faveur des employés des administrations et de commerces.

Ce gain en influence de la fraternité d'assurance parmi le monde ouvrier a-t-il des répercussions à la direction des succursales? Dans quelle mesure, par ailleurs, les directions respectives des Artisans et de l'Alliance se recoupent-elles sur ce plan?

Les chiffres relatifs aux succursales montréalaises montrent des écarts saisissants entre le profil des membres et celui de leurs représentants au cours des années $1910^{20}$. Que ce soit chez les Artisans ou à l'Alliance, les membres ont en effet tendance à confier la direction des affaires locales à trois groupes bien précis : les marchands, les membres des professions libérales et les cols blancs. À eux seuls, ils monopolisent au-delà de $80 \%$ des charges administratives. Sans doute faut-il y voir des groupes prédisposés, par leurs habiletés particulières, à l'exercice de ces fonctions. Mais, la faiblesse de la représentation ouvrière a tout de même de quoi étonner : chez les Artisans, ils ne représentent que $18 \%$ des dirigeants de succursales, alors qu'ils forment pas moins de la moitié des membres du mouve-

20. Les motifs de ce choix sont fort simples. Dans les listes de responsables des succursales publiées régulièrement dans la revue, les adresses civiques des individus sont complètes. Ce qui n'est pas le cas pour les responsables des succursales situées à l'extérieur de l'Île de Montréal. La présence de cette information rend possible le recoupement avec les professions apparaissant dans les bottins d'adresses. 
Tableau 2 - Profil socio-professionnel des dirigeants des succursales montréalaises des Artisans canadiens-français et de l'Alliance nationale, groupe témoin, années 1910 (nombres et \%)* (Sources : compilation personnelle à partir des informations livrées dans les revues L'Alliance nationale et L'Artisan. Les Lovell's Directories ont été utilisés pour le repérage des professions.)

\begin{tabular}{|l|cc|cc|}
\hline \multirow{2}{*}{ Catégories socio-professionnelle } & \multicolumn{2}{|c|}{ artisans } & \multicolumn{2}{c|}{ Alliance nationale } \\
\cline { 2 - 5 } & Nbre & $\%$ & Nbre & $\%$ \\
\hline Manufacturiers/industriels & 0 & 0,0 & 2 & 2,2 \\
Marchands et artisans & 26 & 29,2 & 28 & 30,8 \\
Professions libérales & & & & \\
$\quad$ et personnel hautement qualifié & 11 & 12,4 & 22 & 24,2 \\
Personnel dirigeant & & & & \\
$\quad$ des administrations et entreprises & 8 & 9,0 & 11 & 12,1 \\
Cols blancs semi et non qualifiés & 29 & 32,6 & 19 & 20,9 \\
Cultivateurs & 0 & 0,0 & 0 & 0,0 \\
Cols bleus qualifié et ouvrier de métier & 8 & 9,0 & 6 & 6,6 \\
Cols bleus semi et non qualifiés & 7 & 7,9 & 3 & 3,3 \\
Indéterminés et inclassables & 2 & - & 31 & - \\
\hline Total & 91 & 100,0 & 122 & 100,0 \\
\hline
\end{tabular}

* Comprend les présidents, secrétaires et trésoriers de 33 cercles locaux de l'Alliance nationale et de 29 succursales des Artisans. Afin de permettre la comparaison, les médecins examinateurs (qui siègent d'office dans les cercles de l'Alliance) ont été retranchés. Les professions indéterminées ne sont pas considérées dans le calcul des pourcentages.

ment; à l'Alliance, à peine $10 \%$. L'engagement à la direction de la succursale permet à ces élites d'acquérir une visibilité qui rehausse leur statut dans leur milieu. Leur prestige permet d'asseoir la confiance du milieu dans la mutuelle, de la rendre crédible en quelque sorte. Par un retour des choses, cet engagement leur procure divers bénéfices. Pour le médecin, par exemple, les examens médicaux des candidats à l'assurance et le suivi apporté aux bénéficiaires de la caisse maladie sont une source directe d'honoraires, en plus de les mettre en contact avec des clientèles potentielles. Pour ces petits épiciers, entrepreneurs et boutiquiers, qui évoluent sur le marché local, l'assurance favorise la régulation des rapports de clientèle : " [Le] certificat d'assurance [...], lit-on dans L'Alliance nationale, représente un capital qui assure un crédit. Il représente pour les fournisseurs, médecins, pharmaciens, une certitude qu'ils seront payés ${ }^{21}$."

À la direction centrale du mouvement, dans les deux sociétés d'assurance, le déphasage entre membres et dirigeants est encore plus marqué. À ce niveau précis de l'organisation, points d'ouvriers, ni de cultivateurs, ni d'employés... en somme, une absence à peu près complète de représentation de la grande masse des assurés, comme le montre l'étude qu'Annie Desaulniers a consacrée à l'Alliance nationale ${ }^{22}$. En revanche, se

21. L'Alliance Nationale, "Un aspect trop négligé ", 20, 12 (décembre 1914), p. 1.

22. DESAULNIERS, Annie, op. cit., p. 70-73. 
consolide le pouvoir d'une élite composée, pour l'essentiel, de membres des professions libérales, d'industriels, de marchands, voire, de spécialistes des affaires financières. Loin de s'atténuer, l'écart entre représentants et représentés tend à se creuser entre 1900 et 1945. Et la détention d'un capital de compétences, comme de capital de relations, apparaît de plus en plus comme une condition d'accès aux postes de décision. La direction centrale des Artisans ne paraît pas présenter un profil social très différent ${ }^{23}$.

Du point de vue de son assise sociale, la mutualité d'assurance canadienne-française évolue donc en regard de trois mouvements de fond jusqu'à la Seconde Guerre : l'élargissement de son offre de services aux strates intermédiaires et inférieures du monde ouvrier, une monopolisation des postes de direction locale par les agents de la petite bourgeoisie et le détachement, à la tête de l'organisation, d'une élite " managériale ». Cette triple évolution s'accompagne, pendant l'entre-deux-guerres, d'une redéfinition des rapports de la mutualité au territoire local, là où évoluent les succursales que nous allons maintenant examiner de plus près.

\section{Le déclin des succursales}

Les succursales des Artisans et de l'Alliance nationale ont été le siège d'une vie de relation particulièrement intense à l'échelle des paroisses. Elles sont en effet de toutes les activités commémoratives (religieuses ou patriotiques), délèguent leurs porte-étendard aux cérémonies funéraires, animent des comités de visites aux malades, déploient tout un rituel initiatique pour les recrues, organisent diverses activités à caractère ludique (banquets, pique-nique) à l'intention de la communauté, etc. Toute cette sociabilité locale, à laquelle les revues des deux mutuelles font grand état, sert à cimenter la cohésion du groupe, à entretenir l'intérêt des membres pour leur association et à la rendre visible sur la place publique. Mais, la succursale assume aussi des fonctions économiques capitales. Elle sert d'abord à recruter de nouveaux assurés, une condition vitale dans le commerce d'assurance-vie. Elle exerce par ailleurs des fonctions de surveillance appréciables. Parce qu'à l'échelle locale les membres se connaissent et se reconnaissent, on présume qu'ils sont les mieux placés pour contrôler l'octroi des bénéfices en maladie (en gardant à vue les malades), percevoir les cotisations (en rappelant à l'ordre les retardataires) et écarter les demandeurs d'admission présentant le plus de risques ${ }^{24}$. En définitive, la raison d'être de ces succursales tient pour une large part aux caisses de malades et au recrutement.

\footnotetext{
23. Sur une vingtaine de dirigeants actifs entre 1910 et 1945, on trouve sept médecins, quatre avocats de formation, quatre comptables et un seul ouvrier. Près d'une dizaine d'entre eux sont actifs dans le monde des affaires.

24. "Notre système de caisses des malades ", L'Alliance nationale, 4, 3 (mars 1898), p. 30 .
} 
Sur la base de ce modèle de fonctionnement, les Artisans et l'Alliance ont connu un développement impressionnant : entre 1900 et 1918, le nombre de succursales des deux organisations réunies passe de quelque 225 à plus de 1100 , tandis que le nombre de membres est porté de 20000 à plus de 70000 . Pourtant, à l'approche des années 1920 , on commence à les remettre en question et, pendant la Seconde Guerre, elles sont abandonnées par les promoteurs des deux mutuelles. En 1948, l'Alliance nationale devient une compagnie d'assurance-vie et ses cercles locaux, qu'elle avait déjà remplacés pour la plupart par des " bureaux ", sont relayés par des agences d'assurance. Si les Artisans maintiennent leur filiation juridique au secours mutuel, l'arrivée d'une nouvelle génération de gestionnaires à sa direction, en 1942, se traduit au cours des années qui suivent par des réformes internes non moins importantes. On amende la constitution afin de permettre une augmentation des fonds de la caisse d'administration et d'embaucher de nouveaux spécialistes. On repense également le système de vente, en mettant à contribution des propagandistes " professionnels " rémunérés par le siège social selon les barèmes des compagnies d'assurances.

Pourquoi cet abandon des succursales locales au profit des appareils centralisés? Il s'agit d'une question complexe dont les causes, on s'en doute, tiennent à des facteurs multiples. Écartons tout de suite l'hypothèse de règles de fonctionnement imposées de l'extérieur par l'État : les refontes juridiques étaient souhaitées depuis longtemps par les promoteurs des deux sociétés. L'hypothèse du coup de force d'une élite " managériale " ne résiste pas davantage à l'analyse : il y a certes un renouvellement de la direction centrale au sein des deux sociétés pendant la guerre, mais elle est préparée de longue date, des réformes tout aussi importantes ayant été introduites sous le règne des directions centrales précédentes. Parmi les facteurs qui vont précipiter le déclin des succursales, figurent en tête de liste la défection à l'égard de l'assurance-maladie et la professionnalisation du recrutement. Voyons de plus près.

Dans les deux mutuelles, la gestion des caisses maladie a constitué le tendon d'Achille des succursales locales. La Société des artisans opta très tôt pour la centralisation des fonds d'assurance-maladie dans une caisse unique, en laissant le soin aux succursales de percevoir les cotisations auprès des assurés et d'assurer le suivi des malades. L'Alliance, pour sa part, privilégia à l'origine une décentralisation complète des caisses maladie. Mais, dès 1899, on institua une caisse centrale des malades, espérant sans doute que les dirigeants des succursales finiraient par abandonner la gestion de leurs fonds à l'organisme centralisé. Jusqu'en 1918, alors que les caisses locales sont abolies au bénéfice de l'organisme central, le régime d'assurance-maladie de l'Alliance repose sur cette dualité. Les membres des succursales, semble-t-il, offraient une forte résistance à l'idée d'abandonner la gestion de leurs caisses de malades ${ }^{25}$.

25. C'est ce que suggère la lecture de la revue de l'Alliance qui consacre de nombreux articles à cette question entre 1899 et 1918. Ces articles n'ont cesse de rappeler les 
Figure 4 - Importance relative des revenus générés par les primes d'assurance-maladie, Artisans canadiens-français et Alliance nationale, 1901-1945 (pourcentage du total des revenus en primes)* (Sources : relevé personnel à partir des Rapports annuels du Surintendant des assurances de la province de Québec, des Rapports annuels du Surintendant des assurances du

Canada et des informations livrées dans les revues L'Alliance nationale et

L'Artisan.)

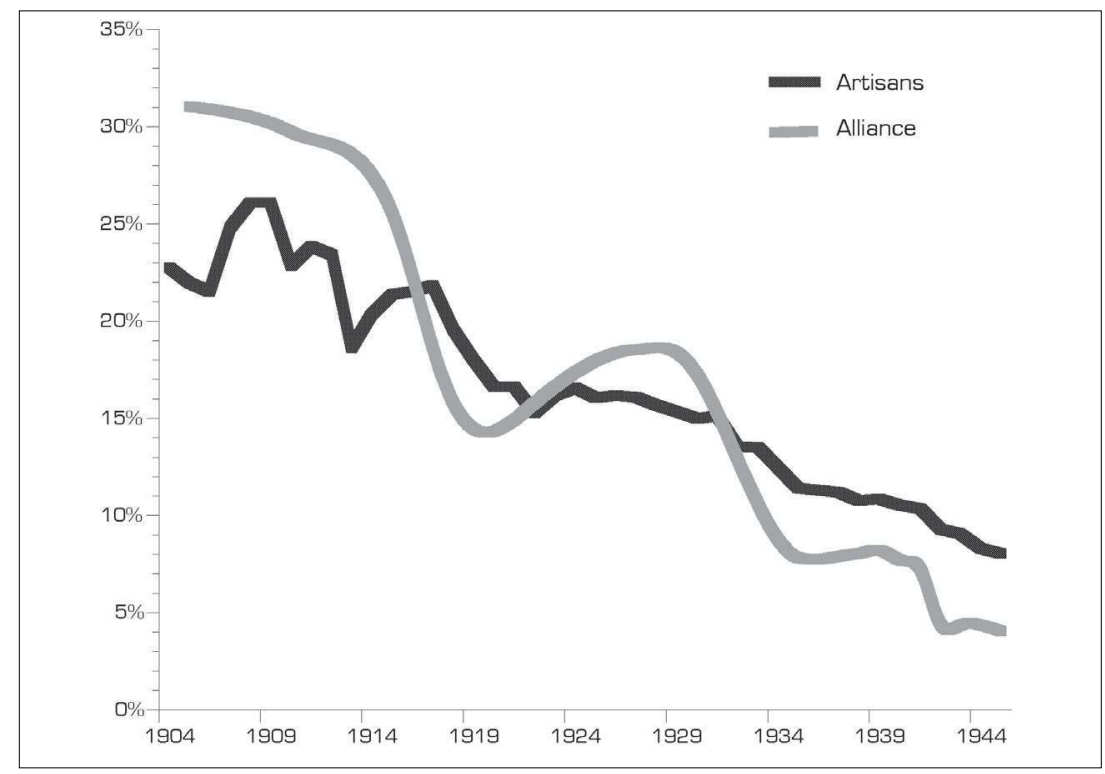

* Pour l'Alliance nationale, les chiffres antérieurs à l'année 1921 sont approximatifs, les informations sur les finances des caisses locales étant déficientes.

$\mathrm{Si}$, au départ, l'assurance maladie est une préoccupation majeure chez les promoteurs des deux mutuelles, elle décline sans cesse au profit de l'assurance-vie, beaucoup plus profitable, qui s'impose rapidement comme la principale base d'affaires des deux mutuelles. Ce détournement, que l'on peut apercevoir à la figure 4, n'est pas propre à l'Alliance et aux Artisans. Il embrasse l'ensemble des sociétés fraternelles. Les historiens ont évoqué plusieurs facteurs pour expliquer le phénomène : l'inadéquation du secours mutuel à la complexité croissante du marché de l'assurance-maladie ${ }^{26}$, la négociation dans les grandes industries et administrations des premiers plans d'assurance collective, l'apparition de sociétés spécialisées dans l'oc-

vertus d'un régime centralisé et, par ricochet, de dénoncer la « négligence " avec laquelle les membres de succursales gèrent les caisses.

26. Si, pendant un certain temps, les visites du médecin à domicile, ou même des comités de visite aux malades des sociétés, pouvaient suffire, les développements de la médecine, de l'hôpital et de l'industrie pharmaceutique rendent de plus en plus inadéquates ces manières de faire. À ce sujet : PETITCLERC, Martin, op. cit. 
troi d'assurance-maladie, l'étatisation de l'assurance contre les accidents du travail et le recours à de nouvelles formes d'épargne pour assurer sa sécurité ${ }^{27}$. Toujours est-il que la désaffection généralisée à l'égard des secours en maladie allait finir tôt ou tard par rendre caduque l'organisation en succursales dans la mesure où c'était là l'une de leurs principales raisons d'être.

Ce désintéressement à l'égard de l'assurance-maladie contraste avec l'énergie sans cesse déployée par les organisations mutuelles pour stimuler le recrutement. Comme on veut offrir cette protection au plus faible coût possible, tout ce travail de mobilisation est orienté vers le membre de la succursale à qui est confiée la responsabilité de convaincre ses proches d'adhérer à l'assurance mutuelle. Il faut dire que sur ce terrain, les sociétés fraternelles subissent une rude concurrence de la part des grandes compagnies qui, depuis le $19^{\mathrm{e}}$ siècle, proposent à leurs clients des polices à rabais, appelées assurance industrielle ou assurance populaire. Le recrutement est sans contredit la préoccupation centrale dans les revues des deux sociétés à l'étude.

Figure 5 - Le recrutement à l'Alliance nationale, selon la provenance des nouveaux membres, 1910-1933 (pourcentage du nombre annuel de demandes d'admissions) (Sources : L'Alliance Nationale, Rapports du président au conseil d'administration, juin 1925 et juin 1934, Rapports annuels, 1901-1934.)

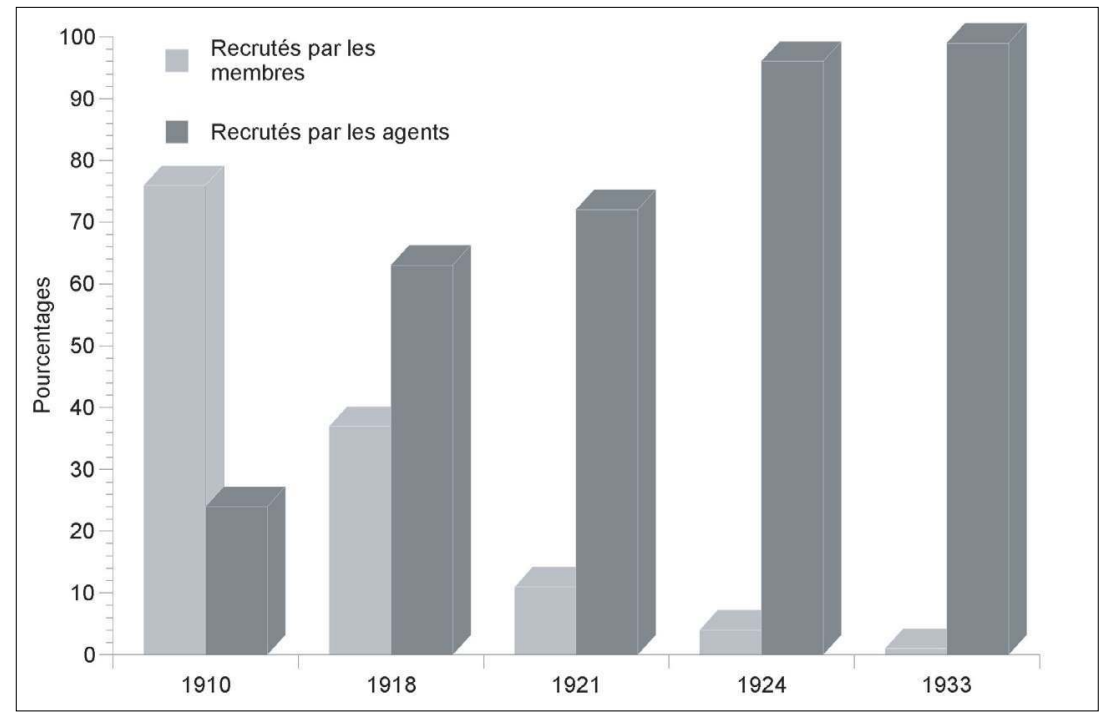

27. Hypothèse soutenue par EMERY, G. et EmerY, J. C., A Young Man's Benefit. The Independant Order of Odd Fellows and Sickness Insurance in the United States and Canada, 1860-1929, Montréal et Kingston, McGill-Queen's University Press, 1999, 184 p. 
Dans quelle mesure les appels à la contribution des membres au recrutement parviennent-ils aux destinataires? Cette contribution connaît tout au long de la période une chute appréciable. La chose a été vérifiée pour l'Alliance nationale (figure 5), mais les données sont manquantes pour le cas des Artisans. Pour la première, les chiffres montrent que l'apport des membres en nouvelles recrues chute radicalement durant les années 1910. Plus on progresse dans le temps, plus s'amenuise l'apport des membres en nouvelles recrues et plus grande est la place laissée à l'activité des professionnels du recrutement. Concours de recrutement, prix aux plus méritants, mots d'ordre, voire menaces à peine voilées, rien n'y fait. Au tournant des années 1920 d'ailleurs, en même temps que l'on centralise les caisses d'assurance-maladie, les dirigeants de l'Alliance lancent une série de réformes dont l'orientation générale ne trompe guère. On cesse de créer des cercles locaux pour privilégier l'organisation de «bureaux " de perception, on procède à l'embauche de " percepteurs " (dont les tâches sont de recueillir les cotisations des membres, d'organiser de nouveaux bureaux locaux et de recruter) et on abandonne la publication de la revue mensuelle.

Du côté de la Société des Artisans, point de réformes aussi précoces et aussi radicales, mais plusieurs indices laissent à penser qu'on assiste là aussi à un fléchissement important de la capacité de recrutement et, d'une façon générale, de la vitalité des succursales. En 1928, le président des Artisans, Rodolphe Bédard, déplorait la disparition de certaines coutumes dans les succursales, rappelant qu'auparavant les commissaires se rendaient spontanément pour apporter le "réconfort d'une visite amicale " aux sociétaires frappés de maladie, [qu'] " on déployait le drapeau des Artisans [lors du décès des membres], [que] les gens se rendaient aux assemblées, [que] le décorum et la procédure étaient respectés. En somme, autrefois, la vie mutualiste était intense ${ }^{28}$ ". Vision idyllique sur les commencements de la mutualité? Sans doute, mais l'intérêt de ce récit sur les " communautés perdues " est de nous renseigner sur la césure entre la succursale et la paroisse, son territoire d'origine. D'ailleurs, avec les années, toute l'activité locale du mouvement, déployée autour des commémorations, comme des activités ludiques et des campagnes de recrutement, tend à s'organiser à l'échelle des " districts ", des regroupements territoriaux de succursales animés par des coordonnateurs appelés " patrons " de districts. Encore à la toute fin des années 1930, les Artisans continuent de valoriser le système de recrutement sans agents d'assurance et les économies administratives qui en découlent ${ }^{29}$. Mais ce système, dont il faut dire qu'il fait appel pour une large part à des " organisateurs et des " recruteurs " à contrat, avait apparemment atteint ses limites. Au sortir de la Guerre, on lui substituera un régime de recrutement centré sur le recours à des propagandistes de métier en prenant soin d'affirmer, devant des critiques de

28. " Nos traditions mutualistes et l'épargne nationale ", L’Artisan, 38, 2 (février 1928), p. 27.

29. L'Artisan, 47, 11-12 (novembre et décembre 1938), p. 5. 
membres faisant valoir que les succursales perdaient ainsi de l'importance, qu'au contraire elles en gagnaient, grâce à « l'aide d'un homme dévoué à l'expansion de la locale ${ }^{30}$ ".

Cette étude exploratoire consacrée à la Société des Artisans et à l'Alliance nationale voulait jeter un éclairage sur les grandes lignes de fracture à travers lesquelles évoluent les grandes sociétés fraternelles jusqu'à la Seconde Guerre. Dans l'entre-deux guerres, les deux mouvements vivent une transition majeure, caractérisée en outre par le passage d'un rapport de sociétariat fortement localisé à un rapport de clientèle. En d'autres termes, leur vie interne se " déterritorialise ", si je peux me permettre l'expression, à la faveur de la consolidation d'un dispositif d'entreprise centralisé pouvant désormais la croissance de la mutuelle sans la contribution active des membres et militants des locales.

Suivant l'histoire particulière de chacune des organisations, cette transition emprunte des sentiers spécifiques, de même qu'elle se réalise à travers des régimes hybrides, fruits des héritages anciens et des contraintes exercées par un marché désormais conditionné par les savoir-faire des grandes compagnies d'assurance-vie. Ces régimes transitoires évoluent à travers une dialectique de la résistance et de l'adhésion à la société assurantielle marchande. Ils s'appuient sur des figures transitoires de l'engagement mutualiste, empruntant à la fois au prototype du " militant " et à celui de l'agent d'assurance contemporain. En lieu et place des membres de succursales, l'Alliance, plus affairiste, optera très tôt pour le " percepteur ", allant jusqu'à saborder sa revue mensuelle, se coupant ainsi d'un important canal de communication entre le bureau central et les cercles locaux. Chez les Artisans, la transition sera plus graduelle, l'" organisateur ", le " recruteur " à contrat et le "membre " ayant longtemps cohabité avant que n'apparaisse le "propagandiste " de métier. La connaissance de ces personnages, des modalités de leur professionnalisation, est la clé pour comprendre la formation du monde assurantiel québécois. Il y a fort à parier que c'est à travers eux que se réalise la poussée mutualiste des années 1880-1939. Cette étude reste à faire.

30. L'Artisan, 57, 5 (mai 1948), p. 13. 


\section{RÉSUMÉ}

Cet article consacré à la Société des Artisans canadiens-français et à l'Alliance nationale cherche à retracer les grandes lignes de fracture à travers lesquelles évolue la fraternité d'assurance canadienne-française jusqu'à la Seconde Guerre mondiale. Partant d'une analyse des bases sociales et territoriales des deux organisations, l'étude met au jour la relation privilégiée qu'elles ont entretenue avec la paroisse, puis l'érosion graduelle de ces liens originels à la faveur de stratégies de croissance à grande échelle. Cette métamorphose de la mutualité d'assurance s'est appuyée sur des figures transitoires de l'engagement mutualiste, empruntant à la fois au prototype du " militant " et à celui de l'agent d'assurance contemporain.

\section{ABSTRACT}

This paper devoted to the Société des Artisans canadiens-français and L'Alliance nationale has undertaken to trace de main lines through which evolves French Canadian mutual aid up to Second World War. Starting from an analysis of the social and territorial bases of both organizations, this study brings up to date the special relationship which they maintained with local parishes, then gradual erosion of these original bonds to the favour of strategies of growth on a large scale. This metamorphosis of mutual aid was concerned by transitory figures, borrowing at the same time from the prototype of "active union member" and that of contemporary insurance agent. 
